\title{
Spin-glass order induced by dynamic frustration
}

\author{
E. A. GOREMYCHKIN ${ }^{1,2}$, R. OSBORN ${ }^{1 \star}$, B. D. RAINFORD ${ }^{3}$, R. T. MACALUSO ${ }^{4}$, D. T. ADROJA ${ }^{2}$ \\ AND M. KOZA 5
}

\author{
Materials Science Division, Argonne National Laboratory, Argonne, Illinois 60439-4845, USA \\ ${ }^{2}$ ISIS Pulsed Neutron and Muon Facility, Rutherford Appleton Laboratory, Chilton, Didcot, Oxfordshire, 0X11 0QX, UK \\ ${ }^{3}$ Department of Physics and Astronomy, University of Southampton, Southampton, S017 1BJ, UK \\ ${ }^{4}$ Program of Chemistry and Biochemistry, University of Northern Colorado, Greeley, Colorado 80639, USA \\ ${ }^{5}$ Institut Laue Langevin, F-38042 Grenoble Cédex, France \\ *e-mail: rosborn@anl.gov
}

Spin glasses are systems whose magnetic moments freeze at low temperature into random orientations without long-range order $^{1}$. It is generally accepted that both frustration and disorder are essential ingredients in all spin glasses, so it was surprising that $\operatorname{PrAu}_{2} \mathrm{Si}_{2}$, a stoichiometric compound with a well-ordered crystal structure, was reported ${ }^{2}$ to show spin-glass freezing. Here, we report on inelastic neutron scattering measurements of crystal-field excitations, which show that $\operatorname{PrAu}_{2} \mathrm{Si}_{2}$ has a singlet ground state and that the exchange coupling is very close to the critical value to induce magnetic order. We propose that spin-glass freezing results from dynamic fluctuations of the crystal-field levels that destabilize the induced moments and frustrate the development of long-range magnetic correlations. This novel mechanism for producing a frustrated ground state could provide a method of testing the concept of 'avoided criticality' in glassy systems.

Frustration arises from competing interactions that favour incompatible ground states ${ }^{1,3}$. For example, in rare-earth intermetallic compounds, the magnetic moments of the $f$ electrons on each rare-earth site interact with neighbouring moments through Ruderman-Kittel-Kasuya-Yosida exchange interactions that oscillate in sign with increasing separation. Except for specific classes of geometrically frustrated lattices, well-ordered crystal structures produce either ferromagnetic or antiferromagnetic order, depending on the energy minimization of the interactions between all the neighbouring moments. However, when there is disorder, in either the site occupations or the exchange interactions, the additional randomness can prevent a unique ordered ground state. Instead, these systems may form spin glasses, which possess a multitude of possible disordered ground states, into one of which the system freezes below the glass transition temperature, $T_{\mathrm{g}}$ (ref. 1). In the thermodynamic limit, spin glasses show broken ergodicity, preventing significant fluctuations to any of the other degenerate spin configurations.

In recent years, two stoichiometric intermetallic compounds have shown evidence of spin-glass order, $\mathrm{URh}_{2} \mathrm{Ge}_{2}$ (ref. 4) and $\mathrm{PrAu}_{2} \mathrm{Si}_{2}$ (ref. 2), both nominally with the same tetragonal ( $\mathrm{ThCr}_{2} \mathrm{Si}_{2}$-type) crystal structure. In both samples, classic spin-glass behaviour was observed $\left(T_{\mathrm{g}}=11 \mathrm{~K}\right.$ and $3 \mathrm{~K}$, respectively), with a frequency-dependent peak in the

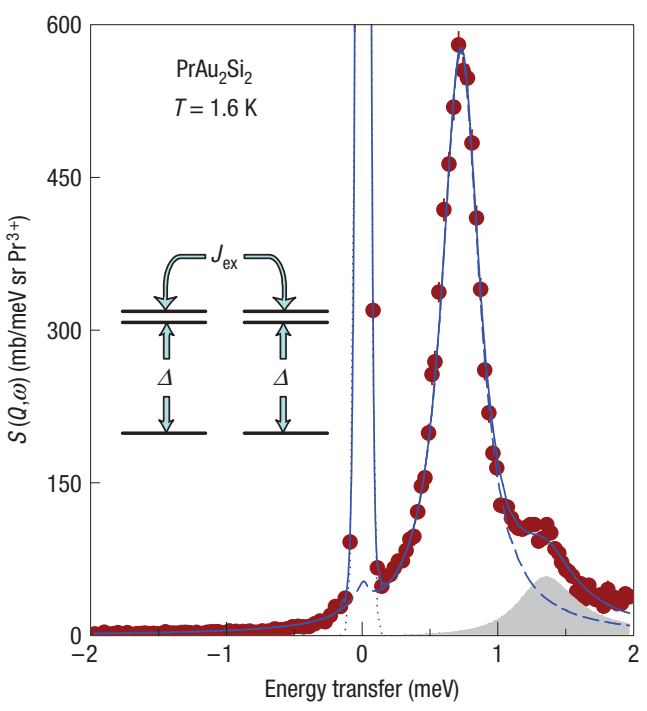

Figure 1 Crystal-field transition in $\mathrm{PrAu}_{2} \mathrm{Si}_{2}$. Inelastic neutron scattering from $\mathrm{PrAu}_{2} \mathrm{Si}_{2}$ measured at $1.5 \mathrm{~K}$ (red symbols) with error bars derived from the square root of the raw data counts. The solid line is a fit to the singlet-doublet crystal-field transition at $\Delta=0.7 \mathrm{meV}$ (dashed line) and an elastic peak from nuclear incoherent scattering (dotted line). The shaded area represents double scattering from the transition at $2 \Delta$. The inset illustrates the mechanism for induced-moment formation, in which interionic exchange coupling, $J_{\mathrm{ex}}$, admixes the excited magnetic doublet into the singlet ground state.

a.c. susceptibility and irreversibility in the field-cooled and zero-field-cooled magnetizations. It was quickly established that spin-glass freezing in the uranium compound resulted from site disorder on the rhodium and germanium sublattices. Extended annealing to remove this source of disorder was sufficient to transform samples into ordered antiferromagnets ${ }^{5}$. On the other hand, the spin-glass behaviour of $\mathrm{PrAu}_{2} \mathrm{Si}_{2}$ is very robust, and appears in the best-quality samples after extensive annealing. 


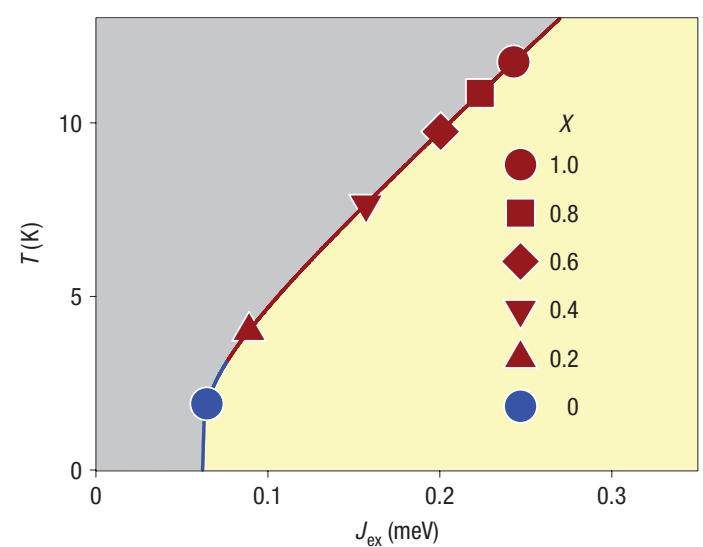

Figure 2 Phase diagram of $\operatorname{PrAu}_{2}\left(\mathrm{Si}_{1-x} \mathrm{Ge}_{x}\right)_{2}$. A self-consistent mean-field calculation of the phase diagram of $\operatorname{PrAu}_{2}\left(\mathrm{Si}_{1-x} \mathrm{Ge}_{x}\right)_{2}$ as a function of the interionic exchange energy, $J_{\text {ex }}$, for a singlet-doublet transition energy, $\Delta=0.7 \mathrm{meV}$. The light area represents the region in which spontaneous magnetic moments are induced by the exchange. The red (blue) symbols are the antiferromagnetic transition (spin-glass freezing) temperatures as a function of $x$. The estimated range of $J_{\mathrm{ex}}$ with spin-glass order is represented by the blue line.
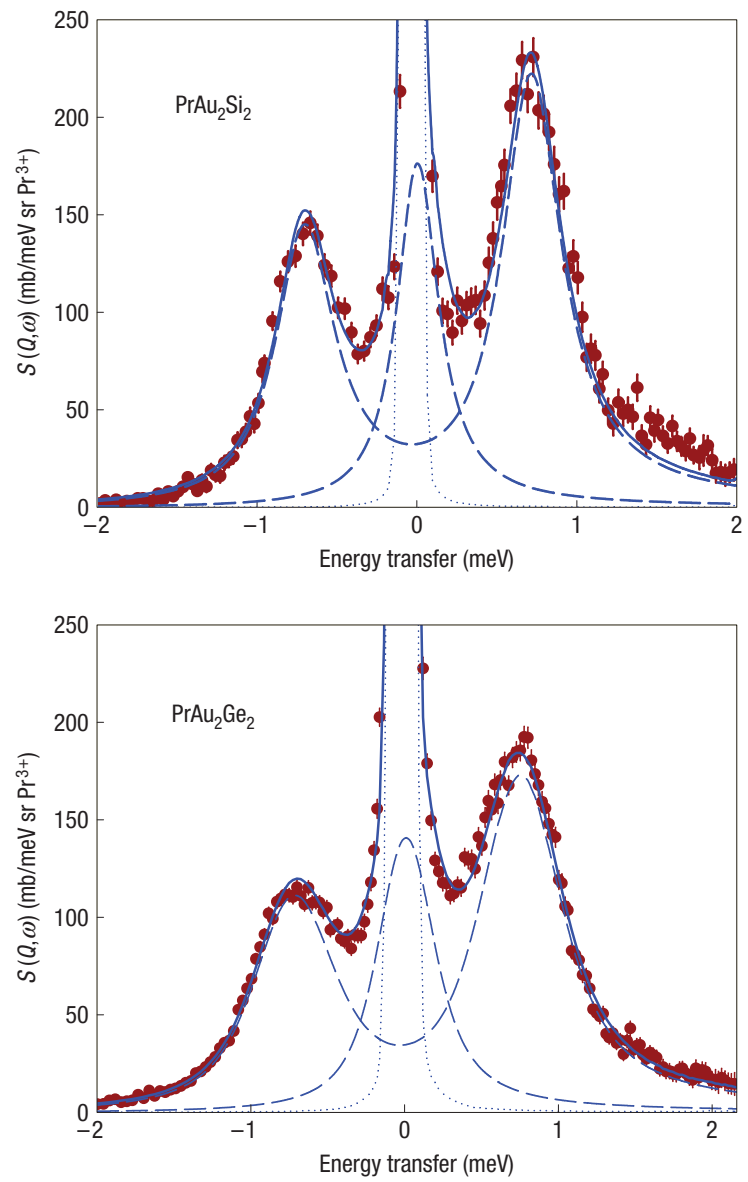

A recent Mössbauer study, combined with neutron and X-ray diffraction, concluded that interchange of gold and silicon atoms was less than 1\% (ref. 6). Furthermore, the intentional introduction of disorder through the substitution of germanium for silicon stabilizes long-range antiferromagnetic order at concentrations greater than $12 \%$ (ref. 7). Finally, the praseodymium sublattice is face-centred tetragonal, and does not contain any of the triangular or tetrahedral motifs normally associated with geometric frustration $^{3}$. This article seeks to explain the origin of magnetic frustration in $\operatorname{PrAu}_{2} \mathrm{Si}_{2}$, given that conventional models involving static disorder or lattice topology do not seem to apply.

First, it is essential to establish the nature of the praseodymium $f$-electron magnetism. The ninefold degeneracy of the $4 f^{2}$-electron states is lifted by the crystal-field potential of tetragonal symmetry produced by the surrounding gold and silicon ions, giving a set of singlet and doublet levels. The doublet states have finite magnetic moments, but the singlet states are non-magnetic, except in the presence of exchange interactions with neighbouring sites as discussed below. Transitions between the crystal-field levels can be observed directly by neutron scattering as inelastic peaks, whose energies and intensities can be used to refine the parameters of the crystal-field potential. The peak widths are inversely proportional to the lifetimes of the excited states, which, in metals, are limited by conduction-electron scattering.
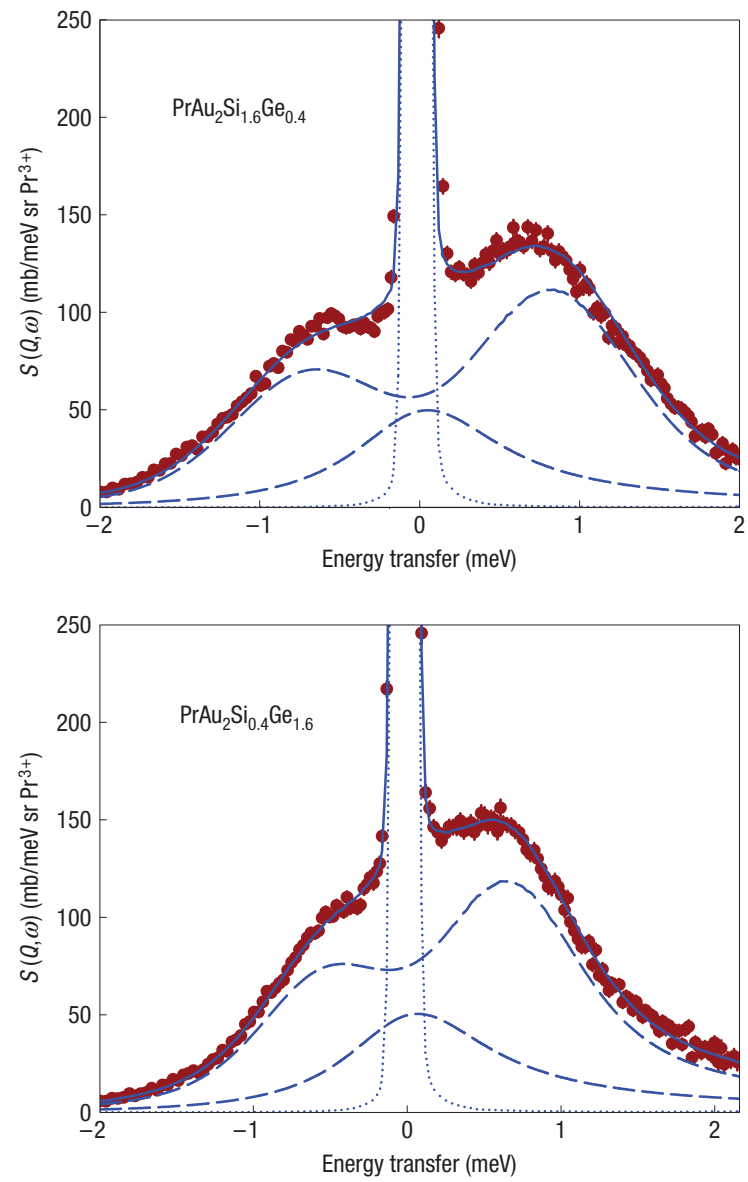

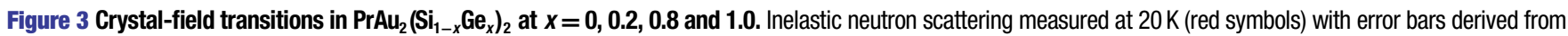
the square root of the raw data counts. The solid line is a fit to three components convolved with the instrumental resolution: two crystal-field components (dashed lines), that is, the inelastic singlet-doublet transition at $\Delta \sim 0.7 \mathrm{meV}$ and the quasielastic scattering from transitions within the excited doublet, broadened by a convolution of Lorentzian and Gaussian lineshapes, and a nuclear incoherent elastic scattering component (dotted line). 
We have used neutron scattering to study samples of $\operatorname{PrAu}_{2}\left(\mathrm{Si}_{1-x} \mathrm{Ge}_{x}\right)_{2}$, with $x=0,0.05,0.10,0.15,0.20,0.80$ and 1, which were synthesized by arc melting followed by annealing in vacuum for four weeks at $850^{\circ} \mathrm{C}$. Neutron-diffraction measurements on $\operatorname{PrAu}_{2} \mathrm{Si}_{2}$ showed no evidence of any additional phases and measurements of the d.c. susceptibility in a field of $20 \mathrm{Oe}$ showed the previously observed difference between field-cooled and zero-field-cooled susceptibilities, confirming a spin-glass freezing temperature of $3 \mathrm{~K}$. The inelastic neutron scattering measurements were carried out at the Institut Laue-Langevin (Grenoble, France) on the high-resolution time-of-flight spectrometer IN6, using an incident energy of $3.1 \mathrm{meV}$, at temperatures ranging from 1.5 to $30 \mathrm{~K}$.

In an earlier report, we determined the phenomenological parameters of the crystal-field potential in $\operatorname{PrAu}_{2} \mathrm{Si}_{2}$ (ref. 8) and showed that the ground state is a non-magnetic singlet with the first excited level a magnetic doublet at an energy $\Delta=0.7 \mathrm{meV}$ (Fig. 1). The remaining levels are all above $7 \mathrm{meV}$, so this is effectively a two-level system at temperatures close to the glass transition.

Before discussing the origin of spin-glass behaviour, we review what is known about magnetic order in singlet ground-state systems. As the ground state of the isolated ion is non-magnetic, a magnetic moment can result only from an admixture of excited crystal-field states produced by exchange interactions with neighbouring praseodymium ions. There is a considerable body of research on such induced-moment systems, both theoretical ${ }^{9,10}$ and experimental ${ }^{11-13}$, showing that, for any given value of the low-lying crystal-field transition energy $(\Delta)$, there is a critical value of the exchange energy $\left(J_{\mathrm{ex}}\right)$. Below this critical value, the system remains paramagnetic (that is, a Van Vleck paramagnet) at all temperatures. However, when the exchange is sufficiently strong, there is a critical temperature below which a ground-state moment forms spontaneously. In a two-level system, this temperature is given in a mean-field model by

$$
T_{\mathrm{c}}=\Delta\left\{\ln \left[\frac{J_{\mathrm{ex}} \alpha^{2}+n \Delta}{J_{\mathrm{ex}} \alpha^{2}-\Delta}\right]\right\}^{-1},
$$

where $\alpha$ is the dipole matrix element coupling the two levels and $n$ is the degeneracy of the excited state. A calculation of $T_{\mathrm{c}}$ as a function of $J_{\text {ex }}$ for $\Delta=0.7 \mathrm{meV}$ and $n=2$ is shown in Fig. 2 .

In most induced-moment systems, $T_{\mathrm{c}}$ marks the transition to long-range order. Examples include $\mathrm{Pr}$ (ref. 12), $\mathrm{Pr}_{3} \mathrm{Tl}$ (ref. 11), $\mathrm{Pr}_{3} \mathrm{In}$ (ref. 14) and $\mathrm{PrNi}_{2} \mathrm{Si}_{2}$ (ref. 13). However, in the presence of static disorder, the low-temperature phase could also be a spin glass, as shown in ref. 15 in a mean-field model developed to explain scandium-terbium alloys ${ }^{16}$ and $\operatorname{PrP}_{0.9}$ (ref. 17). Sherrington ${ }^{15}$ calculated that a sufficiently large distribution of exchange interactions, $\delta J_{\text {ex }}$, could lead to spin-glass freezing. There is a multicritical point at

$$
z J_{\mathrm{ex}}=\sqrt{z} \delta J_{\mathrm{ex}}=\Delta /\left(2 \alpha^{2}\right),
$$

where $z$ is the number of nearest neighbours. At larger values of $\delta J_{\text {ex }}$, spin-glass order would supersede long-range order. In the case of $\operatorname{PrAu}_{2} \mathrm{Si}_{2}$, for which $z=4, \delta J_{\text {ex }}$ should be more than twice $J_{\text {ex }}$, so Sherrington's model requires a high degree of disorder to generate spin-glass behaviour.

Although such strong disorder seems unlikely in $\operatorname{PrAu}_{2} \mathrm{Si}_{2}$, we have explored the Sherrington model further by deliberately disordering the silicon sublattice through germanium substitution. It had already been found that long-range antiferromagnetism in $\operatorname{PrAu}_{2}\left(\mathrm{Si}_{1-x} \mathrm{Ge}_{x}\right)_{2}$ is stabilized at concentrations $x>0.12$ (ref. 7). Our inelastic neutron scattering measurements show that the energy of the singlet-doublet transition is nearly independent of
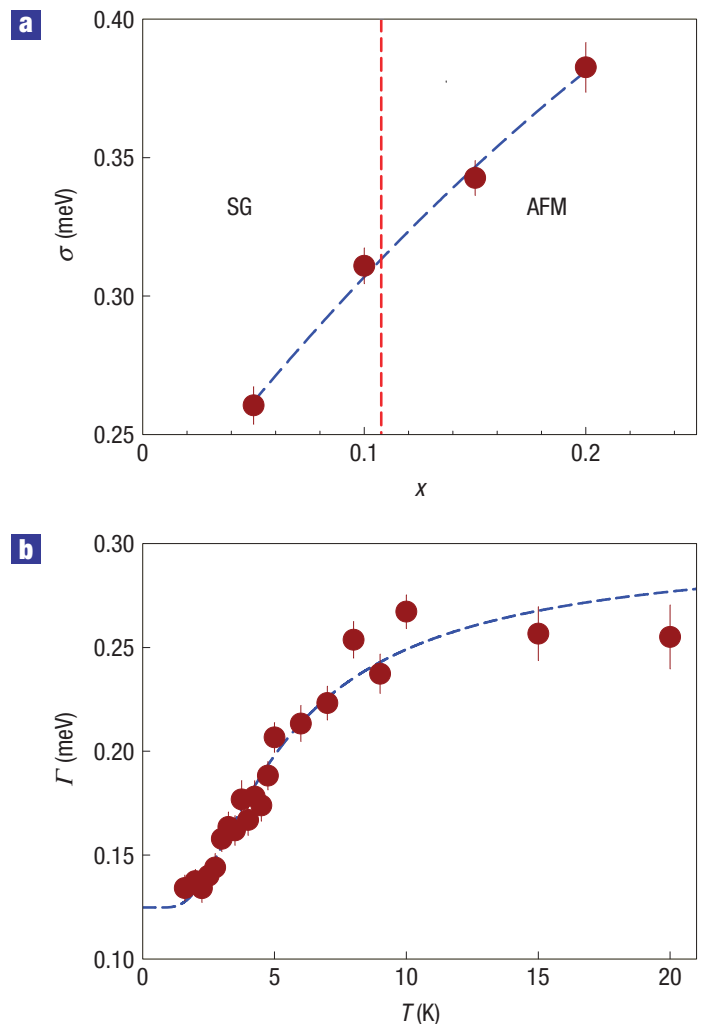

Figure 4 Broadening of the singlet-doublet crystal-field transition. a, The standard deviation $\sigma$ of the Gaussian broadening of the $0.7 \mathrm{meV}$ transition as a function of germanium doping, $x$, measured at $20 \mathrm{~K}$, that is, above $T_{\mathrm{c}}$. The red dashed line marks the critical concentration separating spin-glass from antiferromagnetic order. b. The Lorentzian half-width $\Gamma$ of the $0.7 \mathrm{meV}$ transition in $\mathrm{PrAu}_{2} \mathrm{Si}_{2}$ as a function of temperature. The error bars come from the numerical fits to the peak lineshape described in the legend of Fig. 3 . The blue dashed lines are guides to the eye.

$x$ (Fig. 3). What does change is the strength of the interionic exchange, $J_{\mathrm{ex}}$. In Fig. 2, we estimate the strength of $J_{\mathrm{ex}}$ versus $x$, substituting the measured values of $T_{\mathrm{c}}$ in equation (1). This shows that $J_{\mathrm{ex}}$ increases by a factor of four from $x=0$ to 1 . Germanium doping should therefore be an ideal way of enhancing the exchange disorder that is central to the Sherrington model.

The most noticeable effect of chemical disorder is the large increase in the energy width of the singlet-doublet transition (Fig. 3). The peak lineshape is modelled by the convolution of a Lorentzian and a Gaussian function. The Lorentzian contribution arises from lifetime broadening, which will be discussed later. The Gaussian contribution arises from inhomogeneous broadening due to chemical disorder and the energy dispersion of the crystal-field excitations ${ }^{18}$. There is a small Gaussian contribution in $\mathrm{PrAu}_{2} \mathrm{Ge}_{2}$ $(\sigma=0.13 \mathrm{meV})$ due to energy dispersion, but no Gaussian component is required to fit the data from $\operatorname{PrAu}_{2} \mathrm{Si}_{2}$, whereas the Lorentzian widths are very similar in the two compounds. As the exchange is estimated to be a factor of four smaller in the pure silicide, we believe that chemical disorder dominates the Gaussian contribution at low values of $x$.

If we convolve the peak lineshape with the instrumental resolution to produce the fits in Fig. 3, we estimate that the Gaussian width rises approximately linearly with $x$, for $x \leq 0.2$ (Fig. 4a). This shows that there is no correlation between 

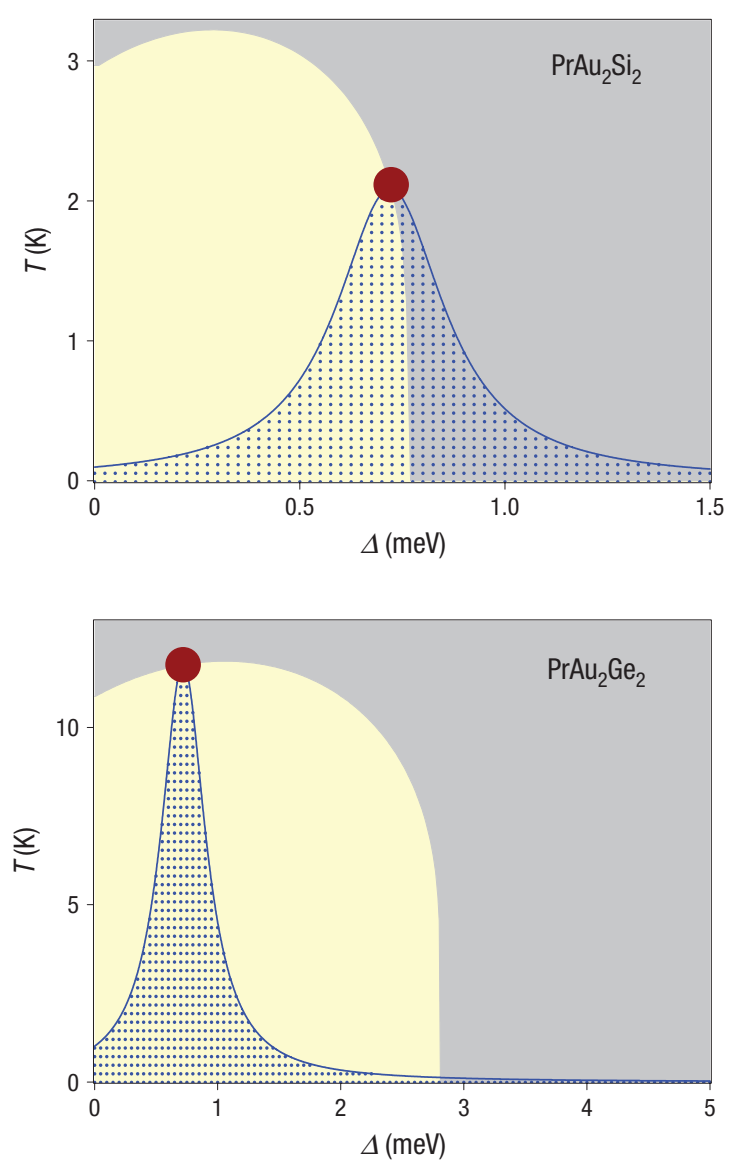

Figure 5 Dynamic instability of induced-moment formation. The mean-field calculation of the phase diagram of $\mathrm{PrAu}_{2} \mathrm{Si}_{2}$ and $\mathrm{PrAu}_{2} \mathrm{Ge}_{2}$ as a function of crystal-field energy $\Delta$ for the respective values of $J_{\text {ex }}$ (Fig. 2). The light areas represent regions of induced-moment stability. The red circle marks the measured singlet-doublet transition energy, and the width of the blue hatched region corresponds to the relaxational broadening of the transition. This illustrates that the induced moment is stable in $\mathrm{PrAu}_{2} \mathrm{Ge}_{2}$ because of the strength of the exchange interactions, whereas it is unstable in $\mathrm{PrAu}_{2} \mathrm{Si}_{2}$ because of its proximity to the phase boundary.

the measured inhomogeneous broadening and the transition to long-range antiferromagnetic order and confirms that static disorder is not the critical factor determining the occurrence of spin-glass freezing.

If static disorder is not responsible for the frustrated ground state, we must seek an alternative explanation, one that should take into account the observation in Fig. 2 that spin-glass freezing occurs only in proximity to the induced-moment phase boundary. We propose that relaxational broadening of the singlet-doublet transition provides the key. Figure $4 \mathrm{~b}$ shows the temperature dependency of the transition half-width in $\operatorname{PrAu}_{2} \mathrm{Si}_{2}$, which increases from about $0.13 \mathrm{meV}$ at $1.6 \mathrm{~K}$ to $0.26 \mathrm{meV}$ at $20 \mathrm{~K}$. The data are consistent with standard models of $f$-electron relaxation due to fluctuations in the single-site crystal-field populations, which causes the rapid increase at low temperatures, and conduction-electron scattering ${ }^{19}$. The important result is that the width remains substantial down to the lowest temperature, with the full-width at half-maximum only falling to a value of $43 \%$ of the transition energy at the glass transition.

This energy broadening will play a significant role in disrupting induced-moment formation. Figure 5 shows the complementary phase diagram to Fig. 2, in which $J_{\text {ex }}$ is fixed to the estimated values in $\operatorname{PrAu}_{2} \mathrm{Si}_{2}$ and $\operatorname{PrAu}_{2} \mathrm{Ge}_{2}$ and $T_{\mathrm{c}}$ is plotted against $\Delta$. The induced moments are predicted to be stable in both compounds; however, if we superimpose the broadened peaks, we see that the peak traverses the phase boundary significantly in the silicon compound but not in the germanium compound. Strictly speaking, the width is proportional to the inverse lifetime of the excited state and not the lifetime of the induced ground state; however, this makes it highly plausible that dynamic fluctuations would disrupt the stability of such moments in $\operatorname{PrAu}_{2} \mathrm{Si}_{2}$, but not in $\operatorname{PrAu}_{2} \mathrm{Ge}_{2}$.

In induced-moment systems, a precursor of long-range order is the appearance in neutron scattering of a quasielastic 'central' peak close to the wavevector of the ordered phase, which is caused by fluctuating regions of short-range magnetic order ${ }^{20}$. For example, in $\mathrm{Pr}_{3} \mathrm{Tl}$, the correlation length associated with this central peak diverges as the critical temperature is approached ${ }^{21}$. Our conjecture is that any divergence of the magnetic correlation length in $\mathrm{PrAu}_{2} \mathrm{Si}_{2}$ is suppressed by dynamic fluctuations that limit the lifetime of induced moments and so introduce magnetic site and exchange disorder. This scenario is similar to the 'avoided criticality' discussed in the theory of structural glass transitions by Tarjus et $a .^{22}$. In their review, they argue that many glasses are close to a conventional second-order phase transition, but that frustration prevents the divergence of the correlation length. This would make the $\operatorname{PrAu}_{2}\left(\mathrm{Si}_{1-x} \mathrm{Ge}_{x}\right)_{2}$ series promising candidates to test their scaling predictions because the degree of frustration can be tuned by varying $J_{\text {ex }}$ or $\Delta$ with dopant concentration or pressure.

There is considerable interest in the ways that spin systems respond to the presence of substantial frustration. Historically, this subject was stimulated by the observation of spin-glass freezing in disordered alloys and compounds, and has more recently focused on systems where the lattice geometry produces a macroscopic degeneracy of possible spin configurations. We suggest that $\mathrm{PrAu}_{2} \mathrm{Si}_{2}$ reveals a new avenue to achieving frustration in systems with neither static disorder nor geometrically frustrated lattices, through dynamic fluctuations, either thermal or quantum, in proximity to a critical phase boundary.

Received 14 February 2008; accepted 18 June 2008; published 27 July 2008.

\section{References}

1. Fischer, K. H. \& Hertz, J. A. Spin Glasses (Cambridge Univ. Press, Cambridge, 1991).

2. Krimmel, A. et al. Spin-glass behavior in $\mathrm{PrAu}_{2} \mathrm{Si}_{2}$. Phys. Rev. B 59, R6604-R6607 (1999).

Ramirez, A. P. Geometric frustration: Magic moments. Nature 421, 483 (2003).

4. Süllow, S. et al. Spin glass behavior in $\mathrm{URh}_{2} \mathrm{Ge}_{2}$. Phys. Rev. Lett. 78, 354-357 (1997).

5. Süllow, S. et al. Disorder to order transition in the magnetic and electronic properties of $\mathrm{URh}_{2} \mathrm{Ge}_{2}$. Phys. Rev. B 61, 8878-8887 (2000).

6. Ryan, D. H. et al. Site disorder and spin-glass ordering in $\mathrm{PrAu}_{2} \mathrm{Si}_{2}$. J. Appl. Phys. 97, 10A908 (2005)

7. Krimmel, A. et al. The evolution from long-range magnetic order to spin-glass behaviour in $\mathrm{PrAu}_{2}\left(\mathrm{Si}_{1-x} \mathrm{Ge}_{x}\right)_{2}$. J. Phys. Condens. Matter. 11, 6991-7003 (1999).

8. Goremychkin, E. A., Osborn, R., Rainford, B. D., Adroja, D. T. \& Koza, M. Magnetic dynamics of the spin-glass system $\operatorname{PrAu}_{2} \mathrm{Si}_{2}$ : An inelastic neutron scattering study. J. Magn. Magn. Mater. 310, 1535-1536 (2007).

9. Grover, B. Dynamical properties of induced-moment systems. Phys. Rev. 140, A1944-A1951 (1965).

10. Cooper, B. R. Magnetic excitons in real singlet-ground-state ferromagnets: Application to $\operatorname{Pr}_{3} \mathrm{Tl}$ and fcc Pr. Phys. Rev. B 6, 2730-2743 (1972).

11. Birgeneau, R. J., Als-Nielsen, J. \& Bucher, E. Magnetic excitons in singlet-ground-state ferromagnets. Phys. Rev. Lett. 27, 1530-1533 (1971).

2. Houmann, J. G. et al. Magnetic excitations and magnetic ordering in praseodymium. Phys. Rev. Lett. 34, 587-590 (1975).

13. Blanco, J. A., Nicklow, R. M. \& Schmitt, D. Paramagnetic excitations in singlet ground state $\mathrm{PrNi}_{2} \mathrm{Si}_{2}$ Phys. Rev. B 56, 11666-11672 (1997).

14. Heiniger, F., Bucher, E., Maita, J. P. \& Longinotti, L. D. Thermodynamic properties of superconductors containing impurities in a crystal-field singlet ground state. Phys. Rev. B 12, 1778-1789 (1975).

15. Sherrington, D. The induced-moment spin glass: Simple mean-field theory. J. Phys. C 12, L929-L933 (1979).

16. Sarkissian, B. V. B. \& Coles, B. R. Spin-glass to Overhauser-alloy transitions in Yt-rare-earth and Sc-rare-earth alloys. Commun. Phys. 1, 17-23 (1976).

17. Yoshizawa, H., Shapiro, S. M., Hasanain, S. K. \& Guertin, R. P. Neutron scattering study of the crystal electric field levels in an induced-moment spin-glass $\operatorname{PrP}_{0.9}$. Phys. Rev. B 27, 448-455 (1983).

18. de Gennes, P. G. Inelastic magnetic scattering of neutrons at high temperatures. J. Phys. Chem. Solids 4, 223-226 (1958)

19. Jensen, J., McEwen, K. A. \& Stirling, W. G. Magnetic excitations in the longitudinally polarized antiferromagnetic phase of praseodymium. Phys. Rev. B 35, 3327-3340 (1987).

20. Lindgård, P.-A. Correlation theory of planar magnets. Phys. Rev. Lett. 50, 690-693 (1983). 
21. Als-Nielsen, J., Kjems, J. K., Buyers, W. J. L. \& Birgeneau, R. J. Observation of a central mode in an exchange-coupled singlet-groundstate system. J. Phys. C 10, 2673-2678 (1977).

22. Tarjus, G., Kivelson, S. A., Nussinov, Z. \& Vlot, P. The frustration-based approach of supercooled liquids and the glass transition: A review and critical assessment. J. Phys. Condens. Matter 17, R1143-R1182 (2005).

Acknowledgements

We thank A. Murani and R. Stewart for scientific discussions and assistance with the ILL experiments. This work was supported by the US Department of Energy Office of Science, under contract

No DE-AC02-06CH11357. R.T.M. is also supported by Research Enhancement Development at UNC.
Author contributions

This project was planned by E.A.G., in collaboration with R.O. and B.D.R. Neutron experiments were carried out by E.A.G., R.O., B.D.R., R.T.M., D.T.A. and M.K., with samples produced by R.T.M. and

data analysis by E.A.G. E.A.G. and R.O. wrote the paper after extensive discussions with the other coauthors.

Author information

Reprints and permission information is available online at http://npg.nature.com/reprintsandpermissions. Correspondence and requests for materials should be addressed to R.O. 